Do Religious Primes Increase Risk Taking? Evidence Against “Anticipating Divine Protection" in Two Preregistered Direct Replications

\author{
Will M. Gervais ${ }^{1}$ \\ Stephanie E. McKee ${ }^{2}$ \\ Sarah Malik ${ }^{1}$
}

1 University of Kentucky Psychology

2 University of Virginia

Contact: Will Gervais, will.gervais@uky.edu 


\begin{abstract}
Do reminders of God encourage people to take more risks? A recent paper (Kupor, Laurin, \& Levav, 2015) reported 9 studies that all yielded statistically significant results consistent with hypothesis. We conducted two large-sample preregistered direct replications (total $N=1104$ ) of studies in this paper (Studies 1a and 1b), and evaluated replicability via 1) statistical significance, 2) a "small telescopes" approach (Simonsohn, 2015), 3) Bayes factors (Gronau, Ly, \& Wagenmakers, 2017), and 4) meta-analyses pooling across original and replication studies. None of these approaches found replicable effects. Combining both original studies and both replications yields strong evidence in support of the null over a default alternative hypothesis, $B F_{01}=11.04$, meaning that the totality of evidence speaks against the possibility that religious primes increase nonmoral risk taking in these designs. This suggests that support for the "anticipating divine protection" hypothesis may be overstated. Preprint https://psyarxiv.com/8f7qd/
\end{abstract}

Keywords: religion; priming; replication; methods 


\title{
Do Religious Primes Increase Risk Taking? Evidence Against “Anticipating Divine Protection" in Two Preregistered Direct Replications
}

\author{
Keep me safe my God, for in you I take refuge.
} $\sim$ Psalms, 16:1

Does thinking of God make believers feel more comfortable taking risks? To the extent that folks believe in a benevolent God who will shelter them from harm, reflecting on God might encourage believers to take more risks, provided the risks are not morally prohibited. That is, people might "anticipate divine protection" and take risks they otherwise might have avoided. A recent Psychological Science article reported 9 studies with statistically significant effects consistent with this possibility (Kupor et al., 2015). We sought to examine the replicability of these findings in two large preregistered direct replications.

Independent replicability is a one aspect, among many (Devezer et al., 2019), of a progressive and cumulative science. Psychology has been leading the way in recent years by emphasizing increasing methodological rigor, including the use of large sample direct replications of prominent findings (e.g., Hagger et al., 2016; Sanchez, Sundermeier, Gray, \& Calin-Jageman, 2017). Of note, meta-science researchers have proposed various criteria for choosing replication candidates. In one such effort to combine these criteria in an a priori principled way_based on ambiguous initial evidence via Bayesian reanalysis, novelty, influence, and feasibility — the "anticipating divine protection" studies emerged as ideal candidates for replication, one of only two top replication candidate papers from a survey of Psychological Science papers (Field et al., 2019). Specifically, Field and colleagues write of this paper: “Our reanalysis of [Kupor et al.'s] results, in conjunction with other methodological and theoretical criteria considerations heavily underlines this replication candidate as a promising 
target, reporting results that are in need of independent corroboration. We recommend a direct, or pure replication, such that the findings exactly as they are presented can be verified."

We elected to replicate the first two studies of the Kupor et al. paper. This paper addresses two related topics that have been at the center of recent methodological controversy: religious priming (Shariff, Willard, Andersen, \& Norenzayan, 2016; Van Elk et al., 2015) and so-called social priming more generally (e.g., Harris, Coburn, Rohrer, \& Pashler, 2013; Payne, Brown-Iannuzzi, \& Loersch, 2016). While there are no known direct replications of the first two studies (those we chose to replicate), some related work does exist (Chan, Tong, \& Tan, 2014), including one direct replication of a different study in the Psychological Science paper that found results contradictory to the original (Gruneau Brulin et al., 2018). Finally, we chose to replicate studies in domains in which we have considerable expertise ${ }^{1}$, hopefully minimizing concerns that any discrepancies between original and replication results stem from lack of expertise or prior knowledge on our end. We were in contact with two of the original paper's authors during study design and implementation, and we thank Drs. Kupor and Laurin for their candor and helpfulness throughout the replication process.

We report how we determined our sample sizes, all data exclusions (if any), all manipulations, and all measures in the studies. Experimental scripts, data, and code are available at https://osf.io/64ct2/

\section{Method}

\section{Replication Study 1a}

\footnotetext{
${ }^{1}$ The first author has even published papers relying on the exact same priming manipulation
} used in the original paper, although he now is skeptical that it does much of anything. 
Participants. We aimed to collect samples at least 2.5 times as large as original studies, and maximized the sample sizes we could acquire given our financial constraints. These constraints led us to fall a little short of our preregistered target of 600 participants per replication: We recruited 566 American participants from MTurk. We excluded 9 participants for failing an attention check and 1 participant for failure to complete the dependent variable, leaving a total sample of $556^{2}$ (304 women, 250 men, 2 unreported). The average age was 36.27 years $(S D=11.40)$. Additionally, $59.9 \%$ of participants reported believing in God. This sample size afforded us power greater than .999 to detect the originally reported effect size $(d=.57)$, and greater than .91 for detecting an effect half as large. Table 1 has demographic summaries.

Procedure. We consulted with the original authors as we designed our studies. We also sent them a copy of our survey and they concurred that it was a high-fidelity replication of their studies. Participants were recruited to an MTurk HIT on word games. After consenting to participate in the study, participants were randomly assigned to either a God $(N=275)$ or control ( $N=281)$ condition, following previous religious priming research (Gruneau Brulin et al., 2018). Participants completed a scrambled sentence priming task in which they were asked to create ten sets of four-word sentences out of five provided words. Importantly, in the God condition, five of the ten provided word strings contained words related to religion (e.g., divine, spirit, God, etc.), while the control condition did not contain such words. After completing the sentence scramble task, all participants then completed the Domain Specific Risk-Taking Scale (DOSPERT: Blais \& Weber, 2006). The DOSPERT is a 40-item scale used to assess risk taking behaviors that asks

\footnotetext{
${ }^{2}$ In our preregistration (https://osf.io/m28xv/register/564d31db8c5e4a7c9694b2c2) we stated that we would filter participants according to their performance on the scrambled sentence task. Communication with the original authors revealed that original data were not screened in this way, so here we deviated from our preregistration to maintain fidelity of the replication attempts.
} 
participants to indicate how likely they are to engage in various behaviors (e.g., Going whitewater rafting at high water in the spring; Taking a skydiving class) on scale from "Extremely unlikely" (1) to "Extremely likely" (5). The DOPSERT is composed of five subscales assessing different types of risks: ethical ${ }^{3}$, financial, health/safety, recreational, and social. The DOSPERT is originally scored with an algorithm including both risk perception and risk taking, but—following Kupor and colleagues (2015)—we simply averaged DOSPERT responses across all subscales, with higher numbers indicating more risk taking. Both manipulation and measure were as used in the original research.

As an active control, participants then completed a conjunction fallacy task (Tversky \& Kahneman, 1983), unrelated to the previous measures. Participants completed the focal tasks with no knowledge that the conjunction fallacy task would follow. In the task, participants learned about "Mary." All participants read: "Mary went to the store and bought tofu, eggplant, broccoli, and frozen meatless lasagna. Is it more likely that Mary is...”. All participants were given the option of choosing a woman, however half of the participants were given a stereotypeconsistent second option (a woman who is a vegetarian), and the other half of participants were given a stereotype-inconsistent second option (a woman who is a big game hunter). Typically, participants are more likely to select the second option for stereotype-consistent targets (vegetarian) than for stereotype-inconsistent targets (big game hunter). This task was merely included to assess whether we could replicate any effects with our participants, and performance on the conjunction task was not treated as diagnostic of anything else. However, if we could not

${ }^{3}$ Although morality and ethics may be dissociable, we note that including ethical risks (e.g., Having an affair with a married man/woman; Not returning a wallet you found that contains $\$ 200$ ) in a measure of nonmoral risk taking may compromise construct validity. Following the original paper, however, we retained these items. 
replicate even a known-replicable phenomenon like the representative heuristic in these samples, we would have concluded that we had a faulty sample, for whatever unknown reason.

Finally, participants answered the following demographic questions: age, gender, belief in God, race/ethnicity, and education level. The full survey is available at https://osf.io/79kqa/ and a preview survey can be completed at

\section{https://virginia.az1.qualtrics.com/jfe/preview/SV_81vV0F11PYzq61v?Q_CHL=preview\&Q_JF}

\section{$\underline{E}=\mathrm{qdg}$}

\section{Replication Study 1b}

Participants. We aimed to collect samples at least 2.5 times as large as original studies, and maximized the sample sizes we could acquire given our financial constraints. Falling slightly short of our preregistered target of 600 participants, we were able to recruit 548 American participants (329 women, 217 men, 2 indicating “Other”) from MTurk. The average age was 36.45 years $(S D=11.73)$. Additionally, $63.5 \%$ of participants reported believing in God. All participants completed the dependent measure. Our sample size gave us power greater than .96 to detect the originally reported effect size $(d=.32)$, but only .46 to detect an effect half that size. Table 1 has demographic summaries.

Procedure. We consulted with the original authors as we designed our studies. We also sent them a copy of our survey and they concurred that it was a high-fidelity replication of their studies. Participants were first asked to describe a recreational risk they had considered taking in the past (for example, hiking a potentially unsafe trail or going down a potentially dangerous ski slope). All participants then completed the same scrambled sentence priming task (Shariff \& Norenzayan, 2007) as in Study 1a, and likewise were randomly assigned to either the God $(N=$ $267)$ or control $(N=281)$ condition. Following this task, participants returned to their recreational 
risk and answered, "What is the likelihood that you will take this risk in the next month?" $(1=$ extremely unlikely, 7 = extremely likely).

Subsequently, as an active control, participants were given a conjunction fallacy (Tversky \& Kahneman, 1983) task which read: "Eric has a career related to finance and he intensely dislikes new technology. He longs for the old days when things were done with paper and relationships were more important. Which of the following statements is more likely?" All participants were given the option of Eric is a senior citizen but were randomly assigned for the other option to be either stereotype-consistent (Eric is a senior citizen who owns a smart phone but rarely uses it) or stereotype-inconsistent (Eric is a senior citizen who is an avid video game player).

Finally, participants answered the following demographic questions: age, gender, belief in God, race/ethnicity, and education level. The full survey is available at https://osf.io/h5n36/ and a preview survey can be completed at https://uky.az1.qualtrics.com/jfe/preview/SV_4MCyK8KuHjdnMqh?Q_SurveyVersionID=curre $\underline{\text { nt\&Q CHL }=\text { preview }}$ 
Table 1: Sample Demographics for Both Replications

\begin{tabular}{|c|c|c|}
\hline Demographics & Replication 1a & Replication 1b \\
\hline $\mathbf{N}$ & 556 & 548 \\
\hline \multicolumn{3}{|l|}{ Gender } \\
\hline Female & 0.55 & 0.60 \\
\hline Male & 0.45 & 0.40 \\
\hline Other & 0 & $>0.01$ \\
\hline Missing Gender & $>0.01$ & 0 \\
\hline \multicolumn{3}{|l|}{ Age } \\
\hline Mean Age (SD) & $36.27(11.40)$ & $36.45(11.74)$ \\
\hline \multicolumn{3}{|l|}{ Belief in God } \\
\hline Believe in God & 0.60 & 0.64 \\
\hline Don't Believe in God & 0.40 & 0.36 \\
\hline \multicolumn{3}{|l|}{ Race/Ethnicity } \\
\hline African American/Black & 0.07 & 0.07 \\
\hline Asian-American/Asian & 0.10 & 0.06 \\
\hline European-American/Anglo & 0.72 & 0.76 \\
\hline $\begin{array}{l}\text { Hispanic- } \\
\text { American/Latino(a) }\end{array}$ & 0.06 & 0.05 \\
\hline $\begin{array}{l}\text { Native } \\
\text { American/American Indian }\end{array}$ & 0.01 & 0.01 \\
\hline Bi-racial/Multi-racial & 0.04 & 0.04 \\
\hline \multicolumn{3}{|l|}{ Education } \\
\hline Less than a HS degree & 0.01 & $>0.01$ \\
\hline High school degree & 0.10 & 0.09 \\
\hline Some college (no degree) & 0.25 & 0.25 \\
\hline 2-year college degree & 0.13 & 0.12 \\
\hline 4-year college degree & 0.37 & 0.37 \\
\hline Masters level degree & 0.10 & 0.15 \\
\hline Doctorate level degree & 0.03 & 0.02 \\
\hline
\end{tabular}

\section{Results}

\section{Replication Results}

In our preregistration (https://osf.io/m28xv/), we defined replication success according to three criteria: 1) producing statistically significant results in the same direction as the original paper, 2) the use of a "small telescopes" approach for assessing the detectability of effects (Simonsohn, 2015), and 3) a Bayes factor analysis comparing a point null hypothesis to an 
alternative hypothesis based on the original studies' reported effect sizes (Gronau et al., 2017). Figure 1 displays raw results.
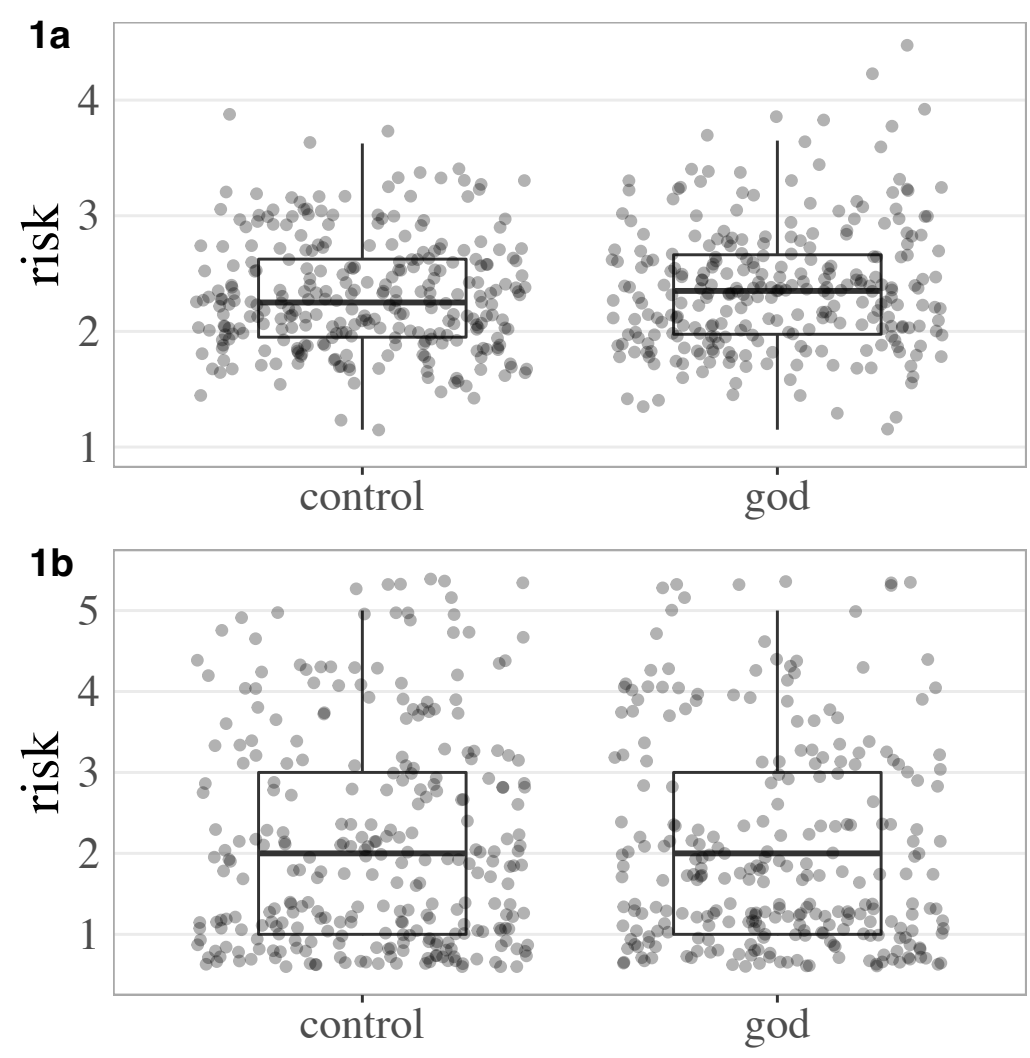

Figure 1. Raw results for Replication 1a and Replication 1b

Statistical significance. Both original studies used two tailed $t$-tests. We followed suit in both replications, using Welch's $t$-tests. Neither replication study yielded directionally consistent statistically significant results, and the second replication was in the opposite direction, $t(544.6)$ $=1.618, p=.106, d=.14[-.03, .30] ;$ and $t(546.0)=-1.320, p=.187, d=-.11[-.28, .06]$, respectively. Neither replication attempt replicated the original findings by this criterion.

Small telescopes. The small telescopes approach assesses the detectability of effects by evaluating the power original studies would have to detect the replication's effect size. It considers a result a "failed" replication if the original studies lacked sufficient power to detect 
the replication result's detected effect size. The sample size of original Study 1a was $N=61$, which would give it $8 \%$ power to detect the replication's effect size (upper bound $=19 \%$ power). The sample size of original Study $1 \mathrm{~b}$ was 202 , yielding power of $12 \%$ (upper bound $=42 \%$ ), although it is important to note that the replication produced directionally opposite results than the original, giving the original study effectively zero power to detect the replication's effect size in a way that would support the original study's described effect. The small telescopes paper recommends a cutoff of $33 \%$ power to assess detectability. Neither replication attempt replicated the original findings by this criterion.

Bayes factors. Bayes factors provide a direct measure of evidence by evaluating the degree to which data support one hypothesis, vis a vis another hypothesis. In psychological research, this typically means pitting a point null hypothesis $(d=0)$ against an alternative hypothesis describing a distribution of plausible effect sizes. All Bayes factor calculations used JASP and the BayesFactor (Morey, Rouder, Jamil, \& Morey, 2015) package in R. For replications, we pitted the null hypothesis against an alternative hypothesis consistent with the effect sizes reported in the original studies. Original Study 1a reported an effect size of $d=.574$ $[.04,1.09]$, so our alternative prior was set to Normal $\sim(M=.574, S D=.267)$. This produced a Bayes factor in which the data support the null, relative to the original study's effect size, by a factor of 3.00; this is generally considered moderate support for the null hypothesis. In an alternative analysis, we used JASP's suggested default priors for the alternative hypothesis (Cauchy, with rscale of .707, one sided in the predicted direction), and found weak support for the null, $\mathrm{BF}_{01}=1.56$. Original Study $1 \mathrm{~b}$ yielded an effect size of $d=.323[.04, .60]$, so our alternative prior was set to Normal $\sim(M=.323, S D=.142)$. This produced a Bayes factor in which the data support the null, relative to the original study's effect size by a factor of 26.13 ; 
this is generally considered strong support for the null hypothesis. In an alternative analysis, we again used JASP's suggested default priors for the alternative hypothesis, and found strong support for the null, $\mathrm{BF}_{01}=23.31$. Neither replication attempt replicated the original findings by this criterion.

Meta-analyses. Finally, as an exploratory addition to our preregistered analyses, we performed mini meta-analyses of the replicated effects. These were meant to synthesize results from the original two studies and our replications, not to summarize all conceptually related work, or to generalize to as-yet-unconducted studies on the topic. We merely wished to summarize the best estimate available from only the original studies and our direct replications. To obtain pooled effect size estimates for each set of studies, we performed two separate random effects meta-analyses (one on original and replication 1a, one on original and replication $1 \mathrm{~b}$ ). Neither yielded pooled effects that significantly differed from zero, $d=.29[-.11, .68], p=.16$; and $d=.09[-.33, .51], p=.66$, respectively. To obtain a pooled effect size estimate including all available data we performed a random effects meta-analysis including all four studies ( 2 original and 2 replication). Again, this meta-analysis revealed a pooled effect size not significantly differing from zero, $d=.17[-.08, .42], p=.18$. Figure 2 summarizes this final model. Neither replication attempt replicated the original findings by this criterion. 


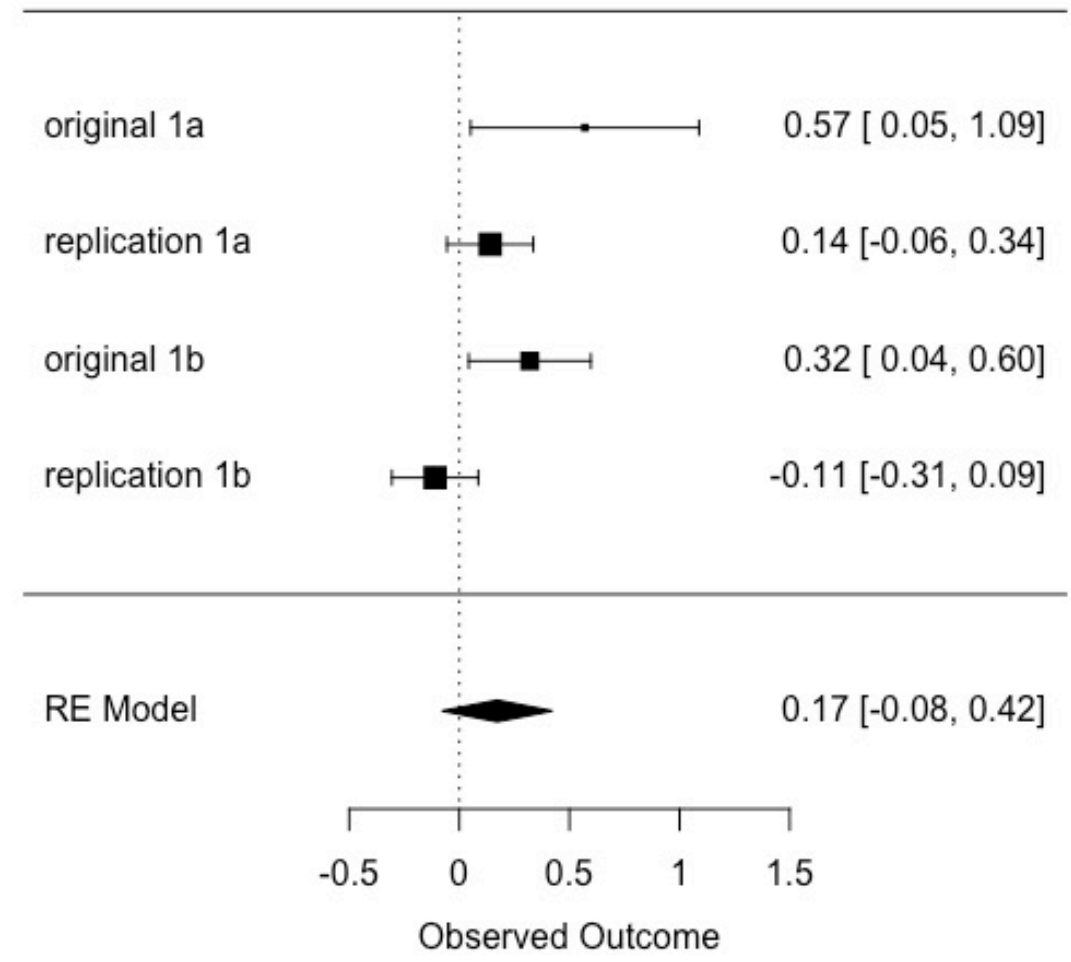

Figure 2. Forest plot of random effects meta-analysis including all available data.

\section{Moderation}

In our preregistration, we specified that we would also probe to see if any priming effects were moderated by participant belief in God, as there is some evidence that religious primes only work among religious believers (Shariff et al., 2016). There was no significant condition by participant belief in God interaction in either replication study, $F(1,552)=.076, p=.783$, and $F(1,544)=.075, p=.784$, respectively

\section{Data Quality and Prime Performance}

Of late, there has been considerable concern over data quality and bot activity on Mechanical Turk. Although the initial studies did not report any screening for data quality or task 
performance, we performed additional exploratory analyses to assess the robustness of any effects among only the most adept participants. Thus, we coded performance on the scrambled sentence task and ran ancillary analyses on only the participants who had zero errors in filling out all 10 scrambled sentences (most participants had errors on one or two sentences). This considerably reduced our effective sample size $(N=164$ and 162 , respectively), but ensured that we only analyzed non-bot participants who exhibited attentiveness, verbal fluency, and maximum engagement with the prime task. In both replication studies, even finely filtered participants failed to support the original studies' inferences: among highly attentive participants, religious priming did not significantly increase nonmoral risk taking, $t(104.9)=.55, p=.59, d=$ $.09[-.24, .42]$, default $B F_{01}=8.14$; and $t(102.6)=-.10, p=.92, d=-.02[-.35, .31]$, default $B F_{01}$ $=5.18$, respectively. Even with the most stringent performance filter, we could not replicate the original effects, and results moderately-to-strongly supported the null relative to a default alternative prior.

\section{Active Controls}

Both replications included conjunction fallacy (Tversky \& Kahneman, 1983) tasks as active controls. They were merely included to see if we could replicate well-known effects in our sample. In both replication studies, participants were significantly more likely to commit conjunction errors for stereotype-consistent targets (a: $>99 \%$; b: 40\%) than for stereotypeinconsistent targets (a: 17\%; b: 1\%), 1a: Odds Ratio $=522$ [161, 3207], $p<2 \times 10^{-16} ; 1 \mathrm{~b}:$ Odds Ratio $=45.4[18.6,150.2], p=1.9 \times 10^{-13}$. We easily replicated a classic paradigm in each replication study, speaking imperfectly to the adequacy of these samples for evaluating basic psychological tasks. The active control was merely included to see if we could replicate a classic effect, and we make no further inferences about it. It is less of a participant attention check and 
more of an MTurk-nihilism check, to see if we can make a classic paradigm work on an overgrazed commons of cheap research participant recruitment.

\section{Discussion}

A 2015 Psychological Science paper (Kupor et al., 2015) presented 9 studies suggesting that religious primes increase nonmoral risk taking. We attempted large preregistered replications of the first two studies from this paper. We could not replicate either study by any of 4 replication metrics. Indeed, Bayesian analyses indicated stronger strength of evidence favoring the null over the initially observed effects in our replications $\left(B F_{01}=3.00\right.$ and 26.13 , respectively) than the original studies had evidence favoring a default alternative hypothesis in the first place $\left(B F_{10}=1.96\right.$ and 1.68 , respectively). Combining both original studies and both replication studies yields strong evidence in support of the null over a default alternative hypothesis, $B F_{01}=11.04$, meaning that the totality of evidence speaks against the possibility that religious primes increase nonmoral risk taking, at least in these designs. Further, a meta-analysis including both original studies and both replications yielded a pooled effect size estimate not significantly different from zero. Combined, this suggests that the original report that God primes increase nonmoral risk taking may be less solid than initially presented.

Why might our pattern of results have diverged somewhat from the initial Psychological Science findings? We attempted to control many potential pitfalls by including active controls, choosing a topic with which we have considerable expertise, and collecting much larger samples from the same population as the original studies (MTurk). That said, it is possible that subtle changes in the makeup of the MTurk population, undetectable to us, may have "killed" these effects (perhaps, for example, through overexposure to similar experiments). Future research on this and related phenomena should provide more stringent tests for participant naivety and 
attentiveness. That said, our results were unchanged-to-bolstered when filtering based on performance on the manipulation task. Although we worked with the original paper's authors to increase the fidelity of our replication, it is possible that some subtle task or recruiting difference - undetected by us or them - compromised our ability to replicate the findings. This possibility, however, speaks against the strength and generality of the phenomenon as initially reported.

Another possibility is that the strength of the original report was simply overstated. The Bayes factors in our replications supported the null more strongly than the initial studies supported the alternative in the first place. The original paper contains 9 statistically significant experiments. But, given modest statistical power inherent to most work in this domain, generating long strings of only significant results is quite improbable (Schimmack, 2012). Further, most of the original $p$-values were more suggestive than strong (Benjamin et al., 2018): key p-values generally ranged between .01 and .05 . This pattern does not appear often in the wild, given genuine effects (Lakens \& Etz, 2017), but is consistent with other similar work that has also faced challenges in replication (Gervais \& Norenzayan, 2012; Sanchez et al., 2017). Perhaps there was never much reason to buy the initially reported effects? At the same time, we note that neither the original studies nor replication attempts conclusively rule out very small effects. The original "anticipating divine protection" paper included results that were consistent with a wide range of effects, including very nearly zero. We merely extend the range of plausibility such that it now also includes zero. It may be worth treating the "anticipating divine protection" hypothesis with skepticism. 


\section{Acknowledgements and Authorship Statement}

All authors designed the studies. Malik and McKee assembled the experimental scripts. Gervais performed analyses and wrote the initial draft. All authors revised the manuscript. We thank students from PSY 440, who also worked with these designs and data as an enlightening pedagogical exercise. 


\section{References}

Benjamin, D. Devezer, B., Nardin, L. G., Baumgaertner, B., \& Buzbas, E. O. (2019). Scientific discovery in a model-centric framework: Reproducibility, innovation, and epistemic diversity. PLOS ONE, 14(5), e0216125. https://doi.org/10.1371/journal.pone.0216125

Field, S. M., Hoekstra, R., Bringmann, L., \& van Ravenzwaaij, D. (2019). When and Why to Replicate: As Easy as 1, 2, 3? Collabra: Psychology, 5(1).

Gruneau Brulin, J., Hill, P. C., Laurin, K., Mikulincer, M., \& Granqvist, P. (2018). Religion vs. The welfare state-The importance of cultural context for religious schematicity and priming. Psychology of Religion and Spirituality, 10(3), 276.

Berger, J. O., Johannesson, M., Nosek, B. A., Wagenmakers, E.-J., Berk, R., . . Camerer, C. (2018). Redefine statistical significance. Nature Human Behaviour, 2(1), 6.

Blais, A.-R., \& Weber, E. U. (2006). A domain-specific risk-taking (dospert) scale for adult populations.

Chan, K. Q., Tong, E. M. W., \& Tan, Y. L. (2014). Taking a leap of faith: Reminders of god lead to greater risk taking. Social Psychological and Personality Science, 5(8), 901-909.

Gervais, W. M., \& Norenzayan, A. (2012). Analytic thinking promotes religious disbelief. Science, 336(6080), 493-496.

Gronau, Q. F., Ly, A., \& Wagenmakers, E.-J. (2017). Informed bayesian t-tests. arXiv preprint arXiv:1704.02479.

Gruneau-Brulin, J., Hill, P. C., Laurin, K., Mikulincer, M., \& Granqvist, P. (2018). Religion vs. The welfare state - the importance of cultural context for religious schematicity and priming. Psychology of Religion and Spirituality, 10(3), 276. 
Hagger, M. S., Chatzisarantis, N. L., Alberts, H., Anggono, C. O., Batailler, C., Birt, A. R., . . . Bruyneel, S. (2016). A multilab preregistered replication of the ego-depletion effect. Perspectives on Psychological Science, 11(4), 546-573.

Harris, C. R., Coburn, N., Rohrer, D., \& Pashler, H. (2013). Two failures to replicate highperformance-goal priming effects. PLoS One, 8(8), e 72467.

Kirkpatrick, L. A., \& Shaver, P. R. (1992). An attachment-theoretical approach to romantic love and religious belief. Personality and Social Psychology Bulletin, 18(3), 266-275.

Kupor, D. M., Laurin, K., \& Levav, J. (2015). Anticipating divine protection? Reminders of god can increase nonmoral risk taking. Psychological Science, 26(4), 374-384.

Lakens, D., \& Etz, A. J. (2017). Too true to be bad: When sets of studies with significant and nonsignificant findings are probably true. Social Psychological and Personality Science, $8(8), 875-881$.

Morey, R. D., Rouder, J. N., Jamil, T., \& Morey, M. R. D. (2015). Package ‘bayesfactor'. URLh http://cran/r-projectorg/web/packages/BayesFactor/BayesFactor pdf i (accessed 1006 15).

Payne, B. K., Brown-Iannuzzi, J. L., \& Loersch, C. (2016). Replicable effects of primes on human behavior. Journal of Experimental Psychology: General, 145(10), 1269.

Sanchez, C., Sundermeier, B., Gray, K., \& Calin-Jageman, R. J. (2017). Direct replication of gervais \& norenzayan (2012): No evidence that analytic thinking decreases religious belief. PLoS One, 12(2), e0172636.

Schimmack, U. (2012). The ironic effect of significant results on the credibility of multiple-study articles. Psychological Methods, 17(4), 551. 
Shariff, A. F., \& Norenzayan, A. (2007). God is watching you priming god concepts increases prosocial behavior in an anonymous economic game. Psychological Science, 18(9), 803809.

Shariff, A. F., Willard, A. K., Andersen, T., \& Norenzayan, A. (2016). Religious priming: A meta-analysis with a focus on prosociality. Personality and Social Psychology Review, 20(1), 27-48.

Simonsohn, U. (2015). Small telescopes: Detectability and the evaluation of replication results. Psychological Science, 26(5), 559-569.

Tversky, A., \& Kahneman, D. (1983). Extensional versus intuitive reasoning: The conjunction fallacy in probability judgment. Psychological Review, 90(4), 293.

Van Elk, M., Matzke, D., Gronau, Q., Guang, M., Vandekerckhove, J., \& Wagenmakers, E.-J. (2015). Meta-analyses are no substitute for registered replications: A skeptical perspective on religious priming. Frontiers in psychology, 6, 1365. 